\title{
Quantification of the Pain and Physical Burden Experienced during Positioning for Craniocaudal Imaging in Mammography, Evaluated by Measurement of Muscle Activity
}

\author{
Mieko Uchiyama ${ }^{1}$, Yongbum Lee ${ }^{1}$, Kiyoko Kazama², Yasuko Minagawa ${ }^{3}$, \\ Masaki Tsurumaki ${ }^{4}$ \\ ${ }^{1}$ Graduate School of Health Sciences, Niigata University, Niigata, Japan \\ ${ }^{2}$ Niigata Hand Surgery Foundation, Niigata Hand Care Center, Niigata, Japan \\ ${ }^{3}$ Kaetsu Comprehensive Health Development Center, Niigata, Japan \\ ${ }^{4}$ Nakajo Central Hospital, Niigata, Japan \\ Email: ${ }^{*}$ uchiyama@clg.niigata-u.ac.jp
}

Received 27 October 2014; revised 9 December 2014; accepted 21 December 2014

Copyright (C) 2015 by authors and Scientific Research Publishing Inc.

This work is licensed under the Creative Commons Attribution International License (CC BY). http://creativecommons.org/licenses/by/4.0/

\section{(c) (i) Open Access}

\begin{abstract}
Mammography is carried out in a special position, i.e. "an image is taken while the breast is compressed, stretched and kept in a fixed position". The purpose of this study was to demonstrate quantitatively and qualitatively the physical and psychological burden due to positioning and breast compression during mammography. Muscle activity of each part of the body during positioning for the standard imaging method, to obtain craniocaudal (CC) view images, was measured in 15 adult females using surface EMG. The associated pain was analyzed using visual analogue scale (VAS) scores. During positioning for the CC view, muscle activity was highest in the biceps (24.44 iEMG/s) followed by the trapezius (17.78 iEMG/s) on the imaging side. Muscle activity of the biceps and the sternocleidomastoid on the imaging side showed significant differences compared with pre-imaging activity (biceps $P<0.031$, sternocleidomastoid $P<0.005$ ). The pain during mammography was rated as moderate to severe pain (VAS = 3.3 - 6.7) for CC views. As a result, the activities of not only the muscles directly involved in mammography positioning but also those indirectly involved were high as compared with the normal state. Measurement of muscle activity during mammography is expected to be used effectively, such as in the care to reduce pain for the subjects.
\end{abstract}

${ }^{*}$ Corresponding author.

How to cite this paper: Uchiyama, M., Lee, Y., Kazama, K., Minagawa, Y. and Tsurumaki, M. (2015) Quantification of the Pain and Physical Burden Experienced during Positioning for Craniocaudal Imaging in Mammography, Evaluated by Measurement of Muscle Activity. Health, 7, 23-34. http://dx.doi.org/10.4236/health.2015.71004 


\section{Keywords}

\section{Mammography, Muscle Activity, Physical Pain, Surface Electromyography, Visual Analogue Scale}

\section{Introduction}

For mammography, the breasts are compressed and extended with a board and the thickness of the breasts is made as thin as possible to enable effective diagnosis and reduce radiation exposure. In addition to breast compression, the neck is bent into an unnatural position with the shoulders also bent, and the posture is maintained for appropriate image imaging [1].

This breast compression and unique positioning are considered a painful and uncomfortable experience for female examinees, although the degree of discomfort varies. Meanwhile, although there is undoubtedly interindividual variability in positioning during mammography due to the experience and knowledge of the radiological technologist, standardization and improvement of the imaging technique have been advanced by systematic certification of screening mammography radiological technologist by the Japan Central Organization on Quality Assurance of Breast Cancer Screening.

Compression of the breasts to make them as thin as possible and unique positioning are required for the examinees. Although the modality requires cooperation by the examinees in this markedly difficult procedure, the pain and physical burden associated with the examination are not clearly understood.

In mammography, advancement of the imaging system, and establishment of appropriate imaging technique and accuracy management have been studied [2]-[9]. Meanwhile, previous reports focusing on the pain and physical burden experienced by the examinees showed that the pain associated with mammography influenced the examinees' behavior when undergoing the screening test [10]-[12], and pain was scored according to the visual analogue scale (VAS) [13]-[19]. These reports suggest that mammography causes a physical burden, in particular, pain.

Meanwhile, the burden on body parts has only been poorly measured and evaluated quantitatively in these reports, although it has been evaluated with subjective markers. Furthermore, the relationship with the burden on body parts other than breasts in mammography has rarely been reported.

In this study, to evaluate the current status of the physical burden and subjective pain associated with mammography, we focused on the electromyogram (EMG) as a physiological parameter. Electromyography has been widely employed for evaluation and analysis of physical movement by extracting the action potential appearing during skeletal muscle contraction as a biological signal [20]-[24]. In particular, it is presumed that a surface EMG, measured with surface electrodes attached to the skin surface, provides data which can be used for quantification of the mammography-associated physical burden.

We previously investigated muscle activity and influence of pain in mediolateral oblique (MLO) imaging, a standard imaging method [25] [26]. The results showed that MLO positioning was associated with increased muscle activity and pain derived from positioning.

This study aimed to clarify the relationship between muscle activity and pain by focusing on muscle activity during positioning for craniocaudal (CC) imaging complementing MLO imaging and quantifying the physical burden experienced by the examinees using surface EMG.

\section{Methods}

\subsection{Systems}

The M-IV (LOARD) mammography system (Hitachi Medical Corporation, Tokyo, Japan) was used in this study. In this study, the examinees were not subjected to X-ray irradiation, because image imaging was unnecessary. Therefore, descriptions of conditions for image imaging are omitted. With regard to compression pressure, the maximum pressure (limiter) was set at 175 N. For measurement of superficial EMG, a polymate AP 1000 (Digix Inc., Tokyo, Japan) was employed. For analysis of the superficial EMG obtained, surface EMG analysis software (NoruPro Light Systems, Inc., Tokyo, Japan) was employed. 


\subsection{Subjects}

The subjects comprised 15 females (in their 30s to 50s) without heart disease, hypertension, or skin disease. Their characteristics were: age, $44.4 \pm 6.6$ years (mean \pm standard deviation); height, $160.0 \pm 6.7 \mathrm{~cm}$; body weight, $5.1 \pm 3.9 \mathrm{~kg}$; and body mass index (BMI), $21.4 \pm 2.2 \mathrm{~kg} / \mathrm{m}^{2}$. They were examined on $14.8 \pm 10.0$ days of the menstrual cycle. Their experience of breast cancer screening was $2.8 \pm 3.2$ times, and five had no experience. The subjects habitually exercised $0.5 \pm 0.5$ times/week but six had no habit of exercise.

These subjects were not those who aimed to undergo screening or investigation but volunteers who had come forward to participate in the study when subjects were recruited. In this paper, the term "imaging" is used throughout the text for convenience but no X-ray irradiation was used in the experiments.

The lack of exposure to radiation was fully explained to the subjects and informed consent was obtained before the start of the study.

Positioning for imaging was adjusted by two female clinical radiological technologist skilled in mammography imaging. Both of them had 15 years' experience of daily work in mammography and one was certified as a screening mammography radiological technologist by the Japan Central Organization on Quality Assurance of Breast Cancer Screening.

\subsection{Subject Muscles}

Muscle activity during positioning was measured from the beginning to the end of mammographic imaging of both breasts. Muscle activity was measured only on the right side of the body to evaluate muscle activity on the imaging side and on the contralateral side. In this paper, muscle activity on the right side of the body during imaging of the right breast and the left breast was defined as "Right" and "Left", respectively.

With a reference of the sites of a severe physical pain according to VAS described by Sharp et al. [18], their related muscles, such as biceps, trapezius, and sternocleidomastoid muscle, as well as gastrocnemius muscle for consideration of the burden on the lower body, were subjected to measurement.

\subsection{Phase Setting in Measurement}

According to the mammographic imaging method, the following phases were set for measurement. The condition before positioning was defined as the relaxation phase (RP). Two phases were defined between the beginning and the end of positioning. The first phase from the beginning of positioning to fixation of the breast was defined as the keep phase (KP). The second phase from the fixation of the breast to the end of imaging was defined as the pressure phase (PP). The measurement protocol is shown in Figure 1. In the order of right breast followed by left breast, positioning for imaging was performed in a standing positon. Before and after positioning of both breasts, the RP was maintained for one minute in a standing position.

\subsection{Measurement Parameters}

\subsubsection{EMG}

In this study, muscle activity was measured by surface EMG. The system used for measurement was the small ambulatory multi-purpose amplifier Polymate AP1000 (Digix Inc.). For an EMG sensor, bipolar active electrodes were placed at one site (Figure 2).

The attachment of the electrodes is shown in Figure 3. Electrodes were attached at the center of the upper edge of the trapezius, which originated from the acromion, and close to the center of the muscle belly of the biceps, sternocleidomastoid muscle, and gastrocnemius muscle. The attachment site was cleaned with the pretreatment compound Skin Pure to reduce contact resistance between the skin and the electrodes before attachment. Furthermore, conductive paste was applied to the electrodes and inductivity was maintained. A grounding wire was attached to the skin of the wrist of all subjects.

\subsubsection{Measurement of Pain and Identification of Painful Body Parts}

Using the VAS for evaluation of pain, the degree of a pain experienced during mammography was measured [27]. A 10-cm straight line was shown and pain was digitalized with a minimum difference of $1 \mathrm{~mm}$. The left end $(0 \mathrm{~cm})$ was defined as "no pain", while the right end $(10 \mathrm{~cm})$ as "the severest pain (the severest pain experienced so far)", and the degree of pain felt at each time-point was indicated by each subject with a finger and 


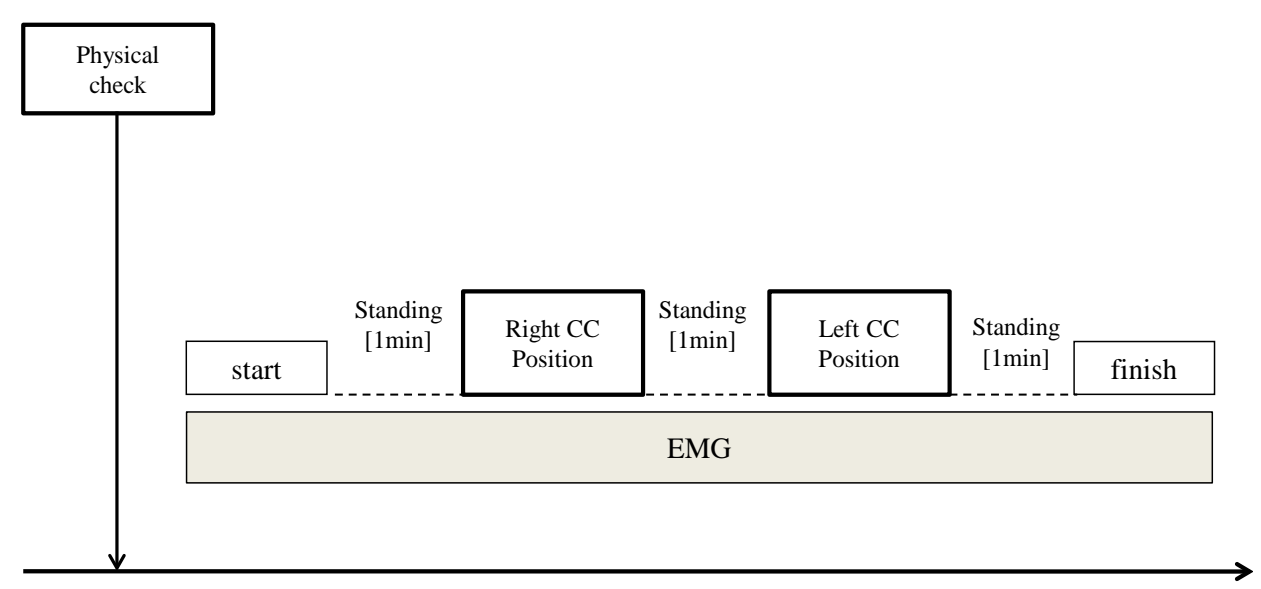

Figure 1. Experimental procedure.

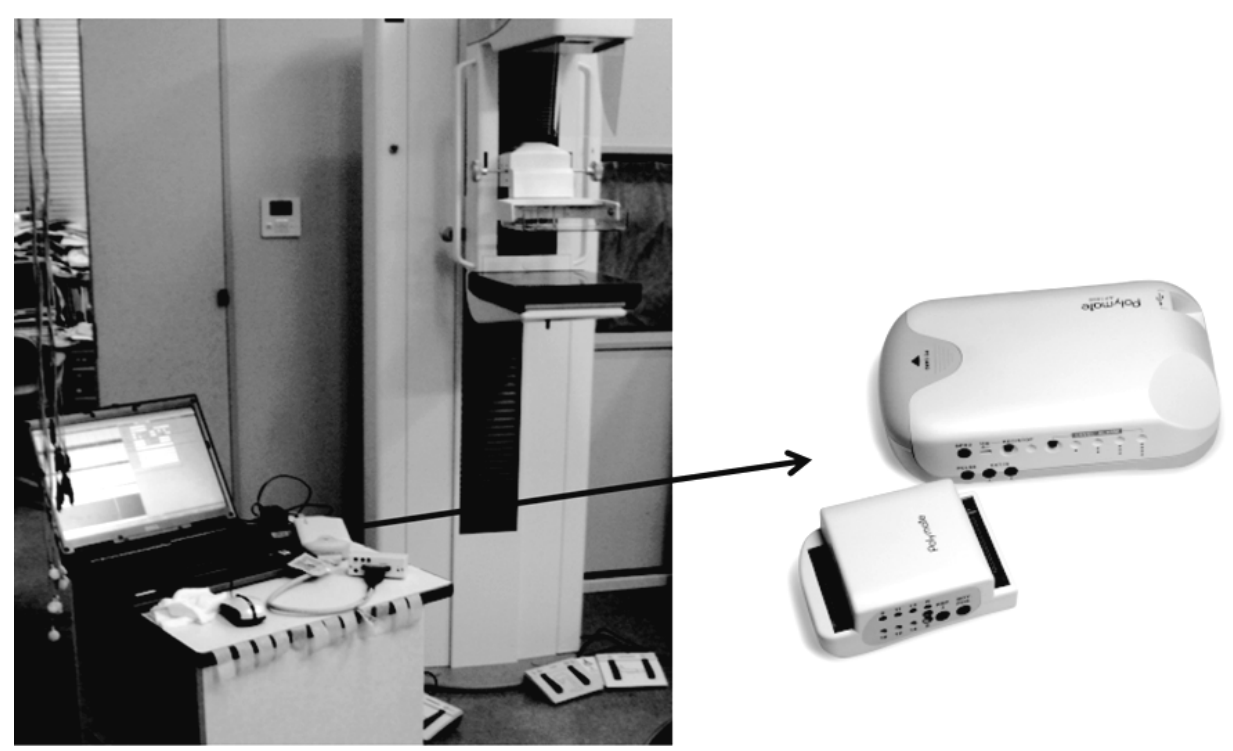

Digital Mammography Equipment HITACHI MEDICAL CORPORATION

Small portable multi-purpose biological amplifier M-IV (LOARD)

Figure 2. Illustrations of mammography equipment and biological amplifier used in this study.

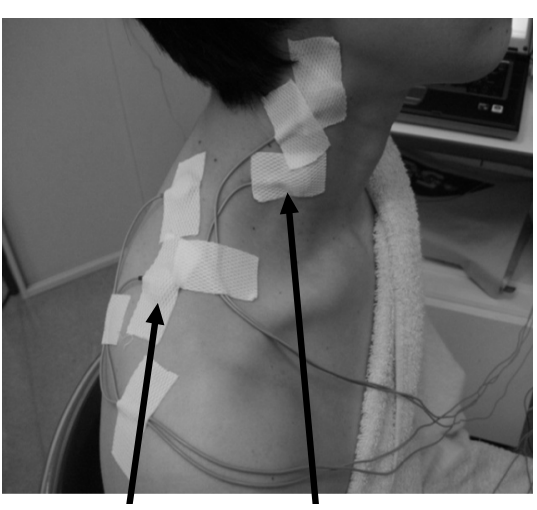

Trapezius \& Sternocleidomastoid

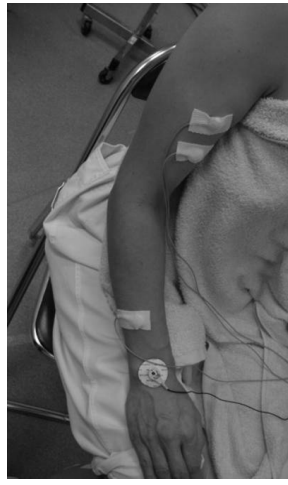

Biceps

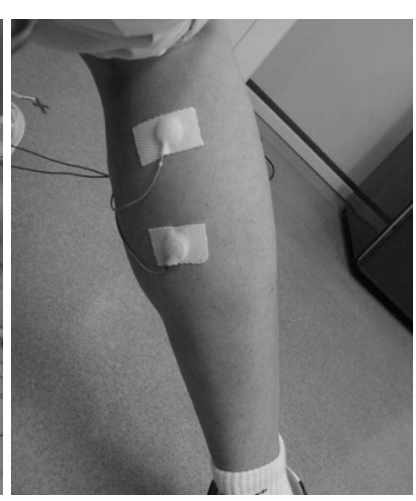

Gastrocnemius

Figure 3. Active EMG sensors placed around the target muscles. 
digitalized. With regard to the parts of the body where pain was felt, the site with the severest pain was selected from an illustration of the anterior and posterior aspects of the whole body and indicated with a finger.

The survey was performed simultaneously with EMG recording and the subjects were asked to pay attention to the degree and the site of pain experienced during imaging. At the end of positioning, they were asked to indicate the degree and the site of the severest pain by pointing to the VAS and the site of pain with a finger.

\subsection{Analytical Method}

\subsubsection{EMG}

The amplitude of EMG waves is dependent on the number of muscle fibers participating in muscle contraction and the voltage derived from them. When muscle activity is increased, the amplitude becomes larger, and when it is decreased, the amplitude becomes smaller. We calculated the value of the integral (iEMG), which equals the sum of the voltages and is proportional to the force of muscle contraction. The measured iEMG was analyzed using a surface EMG analysis program (Surface EMG Analysis, NoruPro Light Systems).

The main frequency zone of EMG signals is $5-100 \mathrm{~Hz}$. However, the amplitude is weak and susceptible to noise. To produce a readable signal, the alternating current noise of iEMG was first removed with a notch filter and a filtering process was performed with a low-frequency blocking filter (blocking frequency, $5 \mathrm{~Hz}$ ) and a high-frequency blocking filter (blocking frequency, $100 \mathrm{~Hz}$ ). The shape of the processed waves was then divided by the length of the defined phases (time, abbreviated as t) for calculation of the mean.

A surface EMG is dependent on the electrode level and may show an inter-examinee, intra-examinee, and inter-muscle difference due to the thickness of subcutaneous fat and skin impedance, even though the voltage occurring at the muscle fiber level is the same. Therefore, EMGs need to be normalized, but the method of normalization remains controversial [21].

In this paper, the condition before positioning or the average of the EMG measurements in the RP was employed as the baseline, which was calculated as the measurement over one min before positioning divided by time.

EMG waves in each phase are shown in Figure 4. With regard to the time of the phase, one min before positioning was defined as $t_{1}$, while the end of positioning was defined as $t_{4}$. The RP was defined as $t_{1}$ to $t_{2}$, with the $\mathrm{KP}$ as $t_{2}$ to $t_{3}$ and the PP as $t_{3}$ to $t_{4}$. Muscle voltage in each phase was calculated as the value of the integral of muscle voltages in each phase (iEMG) divided by the time length of that phase (sec). The value of the integral muscle voltages in the RP or the baseline value was defined as $\mathrm{iEMG}_{r p}$, while that in the KP and PP as $i E M G_{k p}$ and $\mathrm{iEMG}_{p p}$, respectively [Equations (1), (2), and (3)].

$$
\begin{aligned}
\mathrm{iEMG}_{r p} & =\int_{t_{1}}^{t_{2}} \operatorname{EMG}(t) \mathrm{d} t /\left(t_{2}-t_{1}\right) \\
\mathrm{iEMG}_{k p} & =\int_{t_{2}}^{t_{3}} \operatorname{EMG}(t) \mathrm{d} t /\left(t_{3}-t_{2}\right) \\
\mathrm{iEMG}_{p p} & =\int_{t_{3}}^{t_{4}} \operatorname{EMG}(t) \mathrm{d} t /\left(t_{4}-t_{3}\right)
\end{aligned}
$$

The interval between $t_{1}$ and $t_{2}$ is relaxation phase (RP) before positioning. The positioning starts at $t_{2}$. The interval between $t_{2}$ and $t_{3}$ is keep phase (KP). The positioning with breast pressing is completed at $t_{3}$. The interval between $t_{3}$ and $t_{4}$ is pressure phase (PP). The imaging ends at $t_{4}$.

\subsubsection{Comparison of Muscle Activity during Positioning for Imaging}

Measurements in the RP were defined as the baseline and were used to compare measurements in the KP and

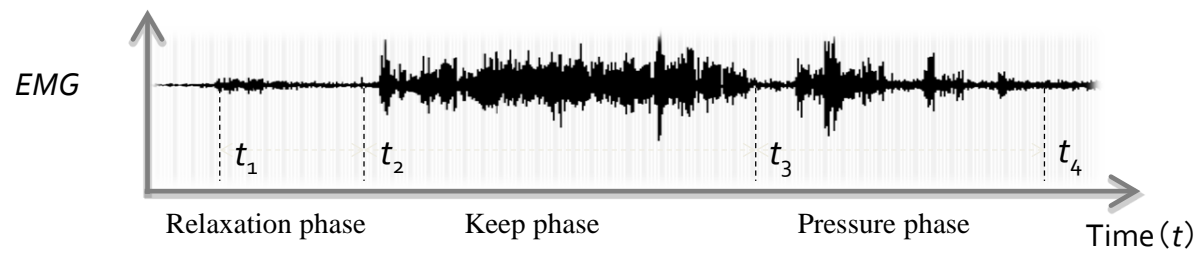

Figure 4. Waves of surface EMG with three phases for analysis. 
PP. One-way analysis of variance (ANOVA) was employed for statistical analysis.

Homoscedasticity was examined and confirmed before statistical analysis using Levene's test. After one-way ANOVA, a comparison was made between the RP and KP or PP. Dunnett's test was carried out for multiple comparisons. The statistical software SPSS 19J for Windows was employed. A P value less than 0.05 was considered statistically significant.

\subsubsection{VAS Scores and Identification of Painful Body Sites}

VAS scores and identification of painful sites were confirmed in the RP, KP, and PP. Data were calculated based on the survey results during positioning on both sides. The same subject was tested twice (subjects [n = 15] $\times 2$ [right and left] = 30). The mean VAS score and the painful sites were examined in each phase.

\section{Ethical Considerations}

The study protocol was submitted in advance to the ethics committee, Health Sciences, Niigata University Graduate School, and approved (approval No. 82).

For consideration, the subjects were fully informed of the significance and aims of the study, detailed experimental methods, measurement methods, potential adverse effect and risk, and predicted treatment in cases of adverse events, and the document explaining all these matters was handed to the subjects. The following was explained to the subjects: participation in the experiment was at the subjects' free will and there would be no disadvantage if consent was not provided; even after consent was given, it could be withdrawn at any time without incurring any disadvantages; and the subjects were free to stop participating in the experiment even after it is had started. Those subjects who consented to the experiment were asked to sign the consent form. Data were identified with numbers so that individual names could not be identified, immediately stored in the flash memory, and processed for analysis on a personal computer that was not connected to the internet. The flash memory, the personal computer used for analysis, and survey sheets were kept in a secure place that could be locked with a key and efforts were made to protect individual information from leakage. It was promised that data would be used only for the study and not for other purposes. After the end of the study, the survey sheet and documents containing the measurement data were cut into pieces and destroyed.

\section{Results}

The mean time for positioning was $55.5 \mathrm{sec}$ for the KP and $10.9 \mathrm{sec}$ for the PP. The pressure of breast compression during positioning was $121.3 \pm 13.5 \mathrm{~N}$ and the thickness after compression was $3.6 \pm 0.60 \mathrm{~cm}$ (Figure 5 ). The results of positioning for imaging were as follows.

\subsection{Muscle Activity in the Different Muscle Groups}

In 15 subjects, the mean \pm standard deviation of the muscle activity on the imaging side and the opposite side is shown in Table 1 . The total muscle activity on the imaging side was: biceps, $19.74 \pm 17.21 \mathrm{iEMG/s}$; trapezius, $13.94 \pm 14.64 \mathrm{iEMG} / \mathrm{s}$; sternocleidomastoid muscle, $13.90 \pm 10.05 \mathrm{iEMG/s}$; and gastrocnemius muscle, $5.20 \pm$ $3.93 \mathrm{iEMG} / \mathrm{s}$.

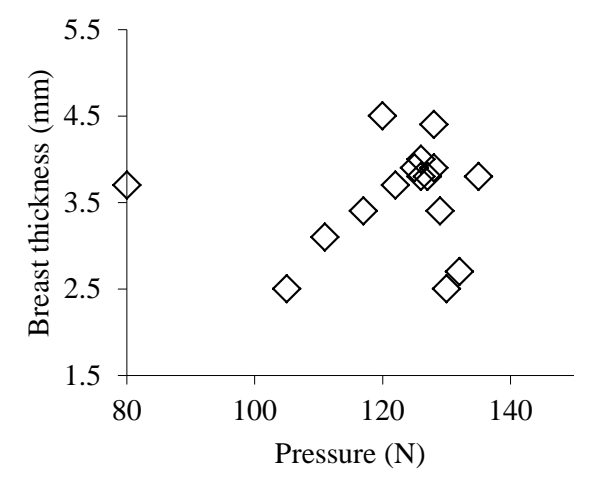

Figure 5. Breast thickness vs. pressure for each subject. 
Table 1. Muscle activities during mammography positioning. $\mathrm{N}=15$ (iEMG/s).

\begin{tabular}{cccccc}
\hline & Muscles & Total $($ Mean \pm SD $)$ & RP $($ Mean \pm SD $)$ & KP $($ Mean \pm SD $)$ & PP $($ Mean \pm SD $)$ \\
\hline \multirow{3}{*}{ Right } & Biceps & $19.74 \pm 17.21$ & $11.51 \pm 14.91$ & $23.28 \pm 17.22$ & $24.44 \pm 17.40$ \\
& Trapezius & $13.94 \pm 14.64$ & $10.74 \pm 20.70$ & $16.82 \pm 12.80$ & $14.26 \pm 7.86$ \\
& Sternocleidomastoid & $13.90 \pm 10.05$ & $8.59 \pm 8.86$ & $15.35 \pm 8.61$ & $17.78 \pm 10.79$ \\
& Gastrocnemius & $5.20 \pm 3.93$ & $3.98 \pm 4.83$ & $6.08 \pm 3.44$ & $5.54 \pm 3.27$ \\
& Biceps & $6.82 \pm 5.85$ & $5.55 \pm 5.46$ & $8.11 \pm 6.61$ & $6.70 \pm 5.44$ \\
\multirow{2}{*}{ Left } & Trapezius & $8.17 \pm 6.46$ & $6.45 \pm 7.63$ & $10.63 \pm 5.77$ & $7.28 \pm 5.42$ \\
& Sternocleidomastoid & $7.90 \pm 4.54$ & $5.67 \pm 3.90$ & $8.73 \pm 3.29$ & $9.24 \pm 5.59$ \\
& Gastrocnemius & $5.66 \pm 3.66$ & $3.74 \pm 3.14$ & $6.66 \pm 3.73$ & $6.51 \pm 3.54$ \\
\hline
\end{tabular}

During the RP, muscle activity on the imaging side was: biceps, $11.51 \pm 14.91 \mathrm{iEMG} / \mathrm{s}$; trapezius, $10.74 \pm$ $20.70 \mathrm{iEMG} / \mathrm{s}$; sternocleidomastoid muscle, $8.59 \pm 8.86 \mathrm{iEMG} / \mathrm{s}$; and gastrocnemius muscle, $3.98 \pm 4.83$ iEMG/s. During the KP, muscle activity was: biceps, $23.28 \pm 17.22 \mathrm{iEMG/s;} \mathrm{trapezius,} 16.82 \pm 12.80 \mathrm{iEMG} / \mathrm{s}$; sternocleidomastoid muscle, $15.35 \pm 8.61 \mathrm{iEMG/s}$; and gastrocnemius muscle, $6.08 \pm 3.44 \mathrm{iEMG} / \mathrm{s}$. During the PP, muscle activity was: biceps, $24.44 \pm 17.40 \mathrm{iEMG/s}$; trapezius, $14.26 \pm 7.86 \mathrm{iEMG/s;} \mathrm{sternocleidomastoid}$ muscle, $17.78 \pm 10.79 \mathrm{iEMG} / \mathrm{s}$; and gastrocnemius muscle, $5.54 \pm 3.27 \mathrm{iEMG} / \mathrm{s}$.

Meanwhile, the total muscle activity on the side opposite the imaging side was: biceps, $6.82 \pm 5.85 \mathrm{iEMG} / \mathrm{s}$; trapezius, $8.17 \pm 6.46 \mathrm{iEMG} / \mathrm{s}$; sternocleidomastoid muscle, $7.90 \pm 4.54 \mathrm{iEMG} / \mathrm{s}$; and gastrocnemius muscle, $5.66 \pm 3.66 \mathrm{iEMG} / \mathrm{s}$. During the RP, muscle activity was: biceps, $5.55 \pm 5.46 \mathrm{iEMG} / \mathrm{s}$; trapezius, $6.45 \pm 7.63$ iEMG/s; sternocleidomastoid muscle, $5.67 \pm 3.90 \mathrm{iEMG} / \mathrm{s}$; and gastrocnemius muscle, $3.74 \pm 3.14 \mathrm{iEMG} / \mathrm{s}$. During the KP, muscle activity was: biceps, $8.11 \pm 6.61 \mathrm{iEMG/s}$; trapezius, $10.63 \pm 5.77 \mathrm{iEMG/s;} \mathrm{sternocleido-}$ mastoid muscle, $8.73 \pm 3.29 \mathrm{iEMG} / \mathrm{s}$; and gastrocnemius muscle, $6.66 \pm 3.73 \mathrm{iEMG} / \mathrm{s}$. During the PP, muscle activity was: biceps, $6.70 \pm 5.44 \mathrm{iEMG} / \mathrm{s}$; trapezius, $7.28 \pm 5.42 \mathrm{iEMG} / \mathrm{s}$; sternocleidomastoid muscle, $9.24 \pm$ $5.59 \mathrm{iEMG} / \mathrm{s}$; and gastrocnemius muscle, $6.51 \pm 3.54 \mathrm{iEMG} / \mathrm{s}$.

The total muscle activities in each group on the imaging side and the opposite side for positioning of CC imaging are shown in Figure 6. Muscle activity of the biceps was higher than that of other muscle groups. Muscle activity was highest in the biceps during the PP and KP on the imaging side, followed by sternocleidomastoid muscle during the PP on the imaging side and trapezius during the KP on the imaging side.

\subsection{Comparison of Muscle Activity during Positioning for Imaging}

Muscle activity was compared between the imaging and the opposite side in each phase. The difference in the mean muscle activity was compared among the different phases and results are shown in Figure 7 (on the imaging side) and Figure 8 (on the contralateral side). One-way ANOVA revealed that the difference in muscle activity on between the imaging side and the contralateral side was significant in both the biceps and sternocleidomastoid muscles (biceps, $\mathrm{F}=3.779$ and $\mathrm{P}<0.031$; and sternocleidomastoid muscle, $\mathrm{F}=5.935$ and $\mathrm{P}<0.005$ ). Multiple comparison analysis revealed a significant difference between the RP and KP in the biceps and between the RP and KP and between the RP and PP in the sternocleidomastoid muscle. These results indicated that muscle activity in the biceps increased during the maintenance of positioning for CC imaging on the imaging side. Moreover, they also suggested that muscle activity in the sternocleidomastoid muscle increased throughout all phases of imaging. One-way ANOVA revealed that there was no significant difference in muscle activity between the different phases on the opposite side to the imaged side in any muscle group. Multiple comparison analysis showed no significant difference in any muscle group between the RP and KP and between the RP and $\mathrm{PP}$. These results indicated that there was no increase in muscle activity in the muscle groups on the opposite side during any phase of positioning for CC imaging.

\subsection{VAS Scores and Identification of Painful Body Sites}

The survey focused on pain experienced during positioning on both sides in each subject. Therefore, the results in a total number of 30 subjects are shown. Table 2 shows mean VAS scores \pm standard deviation. 


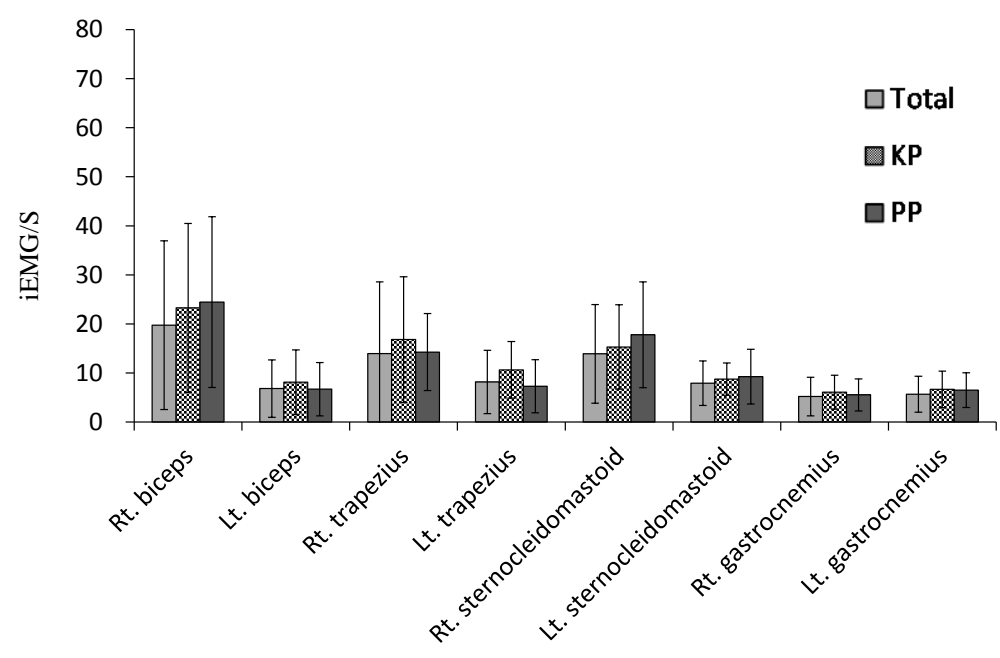

Figure 6. Muscle activities during mammography positioning. Total: Mean of three phase, KP: Keep phase, PP: Pressure phase, Rt.: Right positioning, Lt.: Left positioning.

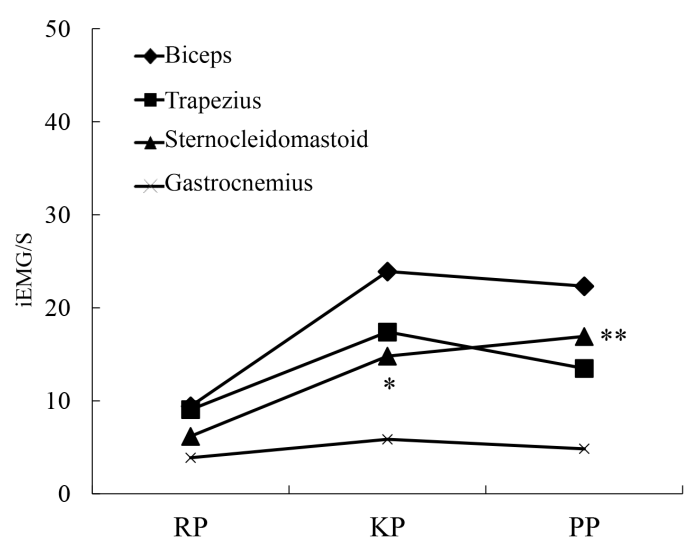

Figure 7. Muscle activities during right breast positioning. All data are expressed as means. ${ }^{* *} \mathrm{P}<0.01$ and $* \mathrm{P}<0.05$ vs. RP values for the same groups.

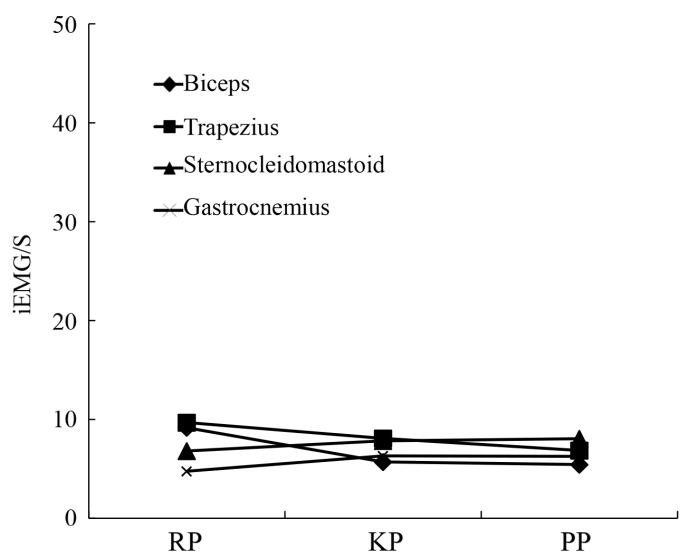

Figure 8. Muscle activities during left breast positioning. All data are expressed as means. ${ }^{* * \mathrm{P}}<0.01$ and $* \mathrm{P}<0.05$ vs. RP values for the same groups. 
Table 2. VAS scores at keep phase and pressure phase. $\mathrm{N}=30$.

\begin{tabular}{cccc}
\hline \multicolumn{2}{c}{ Keep Phase (KP) } & \multicolumn{2}{c}{ Pressure Phase (PP) } \\
\hline For All Data (30) & $3.7 \pm 3.3$ & For All Data (30) & $6.7 \pm 2.8$ \\
Cheek (2) & $6.5 \pm 0.0$ & Cervix (1) & $7.0 \pm 0.0$ \\
Cervix (6) & $3.0 \pm 1.7$ & Breast (25) & $7.4 \pm 2.0$ \\
Shoulder (3) & $3.0 \pm 1.7$ & Waist (2) & $3.5 \pm 0.0$ \\
Breastbone and Rib (3) & $5.0 \pm 1.7$ & Without (2) & $0.0 \pm 0.0$ \\
Breast (7) & $8.1 \pm 0.7$ & & \\
Without (9) & $0.0 \pm 0.0$ & & \\
\hline
\end{tabular}

The numerical value in parentheses indicates the number of trials. Values are presented as means \pm standard deviation.

VAS scores were 1 or higher in 21/30 (70.0\%) subjects in the KP and 28/30 (93.3\%) in the PP. VAS scores were $3.7 \pm 3.3 \mathrm{~cm}$ in the KP and $6.7 \pm 2.8 \mathrm{~cm}$ in the PP. In both phases, the mean VAS score was greater than 3 , which indicated moderate levels of a pain.

With regard to the sites of pain in the KP, the VAS score was $6.5 \pm 0.0 \mathrm{~cm}$ for the cheeks, $3.0 \pm 1.7 \mathrm{~cm}$ in the neck, $3.0 \pm 1.7 \mathrm{~cm}$ at the shoulders, $5.0 \pm 1.7 \mathrm{~cm}$ at the sternum and ribs, and $8.1 \pm 0.7 \mathrm{~cm}$ on for the breasts. In the PP, VAS scores were $7.0 \pm 0.0 \mathrm{~cm}$ in the neck, $7.4 \pm 2.0 \mathrm{~cm}$ for the breasts, and $3.5 \pm 0.0 \mathrm{~cm}$ at the waist area.

Table 2 shows the frequency of pain felt at each site in parentheses. In the KP, VAS scores were positive in $7 / 30$ (23.3\%) for the breasts, 3/30 (10.0\%) at the sternum and ribs, 3/30 (10.0\%) at the shoulders, 6/30 (20.0\%) in the cervix, and 2/30 (6.7\%) for the cheeks. In the PP, VAS scores were positive in 2/30 (6.7\%) at the waist area, 25/30 (83.3\%) for the breasts, and 1/30 (3.3\%) in the cervix. In the KP, meanwhile, VAS scores showed mild pain at all sites other than the breasts, which were near the scanned breast. During the PP, pain was felt strongly at the breast.

\section{Discussion}

\subsection{Muscle Activity during Positioning for Imaging}

Muscle activity for CC imaging was greatest in the biceps on the imaging side, followed by the sternocleidomastoid muscle and the trapezius both on the imaging side in that order. Muscle activity in the biceps and the sternocleidomastoid muscle on the imaging side was clearly increased compared with that before imaging. These results suggested that muscle activity on the imaging side was increased during positioning for CC imaging and that there was an association of the increase in muscle activity between the biceps and the sternocleidomastoid muscle.

According to the guidelines for positioning for CC imaging, "The subjects are asked to stand squarely to the system with the subject breast placed at the middle of the breast supporting platform. They are asked to face the opposite side of imaging [28].” The instructions indicate the position with the cervix rotated as much as possible so that the breast can be placed more easily within the imaging area. The rotation angle of the neck is 0 - 80 degrees and the sternocleidomastoid muscle on the opposite side contracts to achieve rotation of the cervix [28]. During positioning for CC imaging, subjects are asked to rotate the cervix to almost 80 degrees, the maximum possible range of rotation. The characteristics of this positioning are consistent with increased muscle activity in the sternocleidomastoid muscle. Therefore, increased muscle activity in the sternocleidomastoid muscle during positioning for CC imaging can also be explained anatomically.

With regard to the increased muscle activity in the biceps and its relationship to positioning, the guidelines do not include any suggestions such as bending of the biceps. However, the breast supporting platform is placed in front of the subjects and according to the guidelines, "the subjects are instructed to press the medial chest wall tightly to the breast supporting platform so that the medial side of the breast is placed within the X-ray systems without fail [28].” To have the breast on the breast supporting platform, the posture, in which the center of gravity of the entire body is shifted forward, needs to be maintained. Therefore, since maintenance of this posture in a standing position is unstable, it is necessary to support the body trunk using the upper extremities. During 
positioning for CC imaging, subjects support the body by placing the arm on the imaging side around the breast supporting platform. In addition, they use their other arm to grasp the arm part of the platform.

In the present study, it was thought that muscle activity was increased due to bending of the biceps induced by the posture necessary to put the arm on the imaging side around the platform. In cases of MLO imaging, target muscle groups were the auxiliary muscle groups and cooperative increased muscle activity was recognized [27].

However, the two muscle groups that showed increased muscle activity during CC imaging hardly showed any cooperative increase in muscle activity. The sternocleidomastoid muscle is not complementary to the biceps in contraction [29] [30]. Therefore, there is little direct anatomical association between the bending of the biceps and the sternocleidomastoid muscle.

Nevertheless, as the breast was compressed, muscle activity was increased in both the biceps and the sternocleidomastoid muscle and these increases were found to be associated. These results indicated that as breast compression and body fixation increased, muscle activity also increased in the different muscle groups during positioning for CC imaging.

Our results demonstrate that both the biceps and the sternocleidomastoid muscle, which had little association with each other, had mutual influence during positioning for CC imaging. These results suggest that as the breast is compressed, muscle groups that have little cooperative activity also influence each other.

\subsection{Relationship between Positioning for Imaging and Pain}

During the entire mammography procedure, from the beginning of positioning to the compression and fixation of the breast, the mean VAS score at the site where pain was felt was 3 or higher at all sites. These results suggest moderate to severe pain. They also suggest that mammography is accompanied by pain other than that associated with breast compression during CC imaging. Pain was felt in the breasts, neck, shoulders, sternum and ribs, and the cheeks. Since the active muscle groups did not always correspond to the sites of pain, it was impossible to clearly verify the association.

During positioning for CC imaging in the present study, the auricle and head were placed along the fixation plate with the neck rotated to support the rotation of the head. This positioning corresponded to the site of pain. These results suggest the possibility that pain was the result of positioning.

Similarly to MLO imaging, severe pain was felt during the PP and the site of pain was the breast in almost all answers [27]. During the PP, the body was fixed in position with the breast compressed, and this suggested that direct compression was the cause of the pain.

On the other hand, subjects answered that the sites of pain were located near the sites where muscle activity was different during the KP. These included the biceps, sternocleidomastoid muscle, the neck, and the shoulders on the imaging side. This suggests that they influenced not only the pain and burden associated with breast compression directly involved in mammography but also a physical burden on the whole body and a psychological burden continuously present from the time before the screening, and were related to the results we had previously verified [31]-[34].

\section{Conclusions}

In this study, muscle activity was measured at various body sites during mammography by surface EMG. The following results were verified in 15 adult female subjects.

In CC imaging, the biceps on the imaging side (24.44 iEMG/s) showed the highest muscle activity, followed by the sternocleidomastoid muscle (17.78 iEMG/s). There was a significant difference in muscle activity in the biceps and sternocleidomastoid muscle on the imaging side compared with that before imaging (biceps, $\mathrm{P}<$ 0.031; and sternocleidomastoid muscle, $\mathrm{P}<0.005$ ). The pain during imaging in mammography was moderate to severe (VAS, 3.3 - 6.7).

\section{Acknowledgements}

This work was supported by JSPS KAKENHI Grant Numbers 23593285, 26463372.

\section{References}

[1] Henrich, R.E., Baett, L., Botsco, M.A., Deibei, D., Feig, S. and Gray, J. (1999) Mammography Quality Control Manual: 
Patient Positioning and Compression. American College of Radiology, 30-75.

[2] Haus, A.G. (2002) Historical Technical Developments in Mammography. Technology in Cancer Research \& Treatment, 1, 119-126. http://dx.doi.org/10.1177/153303460200100204

[3] Ng, K.H. and Muttarak, M. (2003) Advances in Mammography Have Improved Early Detection of Breast Cancer. J. HK. Coll. Radiol., 6, 126-131.

[4] Ng, K.H., Jamal, N. and DeWerd, L. (2006) Global Quality Control Perspective for the Physical and Technical Aspects of Screen-Film Mammography_-Image Quality and Radiation Dose. Radiation Protection Dosimetry, 121, 445-451. http://dx.doi.org/10.1093/rpd/ncl051

[5] Yamada, T. (2010) Current Status and Issues of Screening Digital Mammography in Japan. Breast Cancer, 17, 163168. http://dx.doi.org/10.1007/s12282-009-0191-Z

[6] Thomassin-N.I., Perrot, N., Dechoux, S., Ribeiro, C., Chopier J. and de Bazelaire, C. (2014) Added Value of OneView Breast Tomosynthesis Combined with Digital Mammography According to Reader Experience. European Journal of Radiology, 84, 235-241. http://dx.doi.org/10.1016/j.ejrad.2014.10.022

[7] Cederström, B. and Fredenberg, E. (2014) The Influence of Anatomical Noise on Optimal Beam Quality in Mammography. Medical Physics, 41, Article ID: 121903. http://dx.doi.org/10.1118/1.4900611

[8] Hendrick, R.E., Helvie, M.A. and Hardesty, L.A. (2014) Implications of CISNET Modeling on Number Needed to Screen and Mortality Reduction with Digital Mammography in Women 40-49 Years Old. American Journal of Roentgenology, 203, 1379-1381. http://dx.doi.org/10.2214/AJR.14.12646

[9] Lee, C.I., Cevik, M., Alagoz, O., Sprague, B.L., Tosteson, A.N., Miglioretti, D.L., Kerlikowske, K., Stout, N.K., Jarvik, J.G., Ramsey, S.D. and Lehman, C.D. (2014) Comparative Effectiveness of Combined Digital Mammography and Tomosynthesis Screening for Women with Dense Breasts. Radiology, 30 May 2014, Article ID: 141237. http://dx.doi.org/10.1148/radiol.14141237

[10] Molina, Y., Beresford, S.A., Espinoza, N. and Thompson, B. (2014) Psychological Distress, Social Withdrawal, and Coping Following Receipt of an Abnormal Mammogram among Different Ethnicities: A Mediation Model. Oncology Nursing Forum, 41, 523-532. http://dx.doi.org/10.1188/14.ONF.523-532

[11] Lee-Lin, F., Domenico, L.J., Ogden, L.A., Fromwiller, V., Magathan, N., Vail, S. and Gorman, P.N. (2014) Academic-Community Partnership Development Lessons Learned: Evidence-Based Interventions to Increase Screening Mammography in Rural Communities. Journal of Nursing Care Quality, 29, 379-385. http://dx.doi.org/10.1097/NCQ.0000000000000071

[12] Purtzer, M.A. and Overstreet, L. (2014) Transformative Learning Theory: Facilitating Mammography Screening in Rural Women. Oncology Nursing Forum, 41, 176-184. http://dx.doi.org/10.1188/14.ONF.176-184

[13] Kornguth, P.J., Keefe, F.J. and Conaway, M.R. (1996) Pain during Mammography: Characteristics and Relationship to Demographic and Medical Variables. Pain, 66, 187-194. http://dx.doi.org/10.1016/0304-3959(96)03057-6

[14] Sapir, R., Patlas, M., Strano, S.D., Hadas-Halpern, I. and Cherny, N.I. (2003) Does Mammography Hurt? Journal of Pain and Symptom Management, 25, 53-63. http://dx.doi.org/10.1016/S0885-3924(02)00598-5

[15] Brett, J., Bankhead, C., Henderson, B., Watson, E. and Austoker, J. (2005) The Psychological Impact of Mammographic Screening: A Systematic Review. Psychooncology, 14, 917-938. http://dx.doi.org/10.1002/pon.904

[16] Gram, I.T., Lund, E. and Slenker, S.E. (1990) Quality of Life Following a False Positive Mammogram. British Journal of Cancer, 62, 1018-1022. http://dx.doi.org/10.1038/bjc.1990.430

[17] Taguchi, R., Yamazaki, Y. and Nakayama, K. (2010) Eliciting Preferences for Mammography: Using a Discrete Choice Experiment Approach. Japanese Journal of Public Health, 57, 83-94.

[18] Sharp, P.C., Michielutte, R., Freimanis, R., Cunningham, L., Spangler, J. and Burnette, V. (2003) Reported Pain Following Mammography Screening. Archives of Internal Medicine, 163, 833-836. http://dx.doi.org/10.1001/archinte.163.7.833

[19] Hagen, S., Goodwin, E. and Sinclair, L. (2008) Sitting vs. Standing during Screening Mammography. Radiologic Technology, 79, 214-220.

[20] De Luca, C.J. (2002) Surface Electromyography: Detection and Recording. DelSys Incorporated, 2-10.

[21] Kizuka, T., Masuda, T., Kiryu, T. and Sadoyama, T. (2006) Biomechanism Library Practical Usage of Surface Electromyogram. Tokyo Denki University Press, Tokyo.

[22] Jeitler, M., Brunnhuber, S., Meier, L., Lüdtke, R., Büssing, A., Kessler, C. and Michalsen, A. (2014) Effectiveness of Jyoti Meditation for Patients with Chronic Neck Pain and Psychological Distress-A Randomized Controlled Clinical Trial. The Journal of Pain, 3, 1-28. http://dx.doi.org/10.1016/j.jpain.2014.10.009

[23] Watanabe, M., Kaneoka, K., Wada, Y., Matsui, Y. and Miyakawa, S. (2014) Trunk Muscle Activity with Different Sitting Postures and Pelvic Inclination. Journal of Back and Musculoskeletal Rehabilitation, 27, 531-536. 
[24] Maekawa, Y., Shiozaki, A. and Majima, Y. (2012) A Study on Visualizing the Twist and the Load on the Lumbar Region in Nursing Care. Nursing Informatics, 23, 1-5.

[25] Uchiyama, M., Lee, Y., Kazama, K., Minagawa, Y., Tsai, D.Y., Sadakata, M. and Sayama, M. (2011) A Preliminary Study on Measurement of Muscle Activity during Mammography Positioning. Japanese Journal of Radio-Logical Technology, 67, 679-682. http://dx.doi.org/10.6009/jirt.67.679

[26] Uchiyama, M., Lee, Y., Sadakata, M., Sayama, M. and Tsai, D.Y. (2012) Measurement of Muscle Activities for Evaluating Physical Burden and Pain during Mammography Positioning. The Tohoku Journal of Experimental Medicine, 228, 53-58. http://dx.doi.org/10.1620/tjem.228.53

[27] Dinash, G., Daniel, K., Len, G. and John, G. (2001) Examining the Validity of Pressure Ulcer Risk Assessment Scales: Developing and Using Illustrated Patient Simulations to Collect the Data. Journal of Clinical Nursing, 10, 697-706. http://dx.doi.org/10.1046/j.1365-2702.2001.00525.x

[28] Japan Radiology Congress, Japanese Society of Radiological Technology (2010) Mammography Guidelines. Third Edition, Igakusyoin, Tokyo, 7-13.

[29] Boullosa, D.A., Hautala, A.J. and Leicht, A.S. (2014) Introduction to the Research Topic: The Role of Physical Fitness on Cardiovascular Responses to Stress. Frontiers in Physiology, 5, 1-2. http://dx.doi.org/10.3389/fphys.2014.00450

[30] Bernard, K. and Adachi, K. (2008) Understanding Muscles: A Practical Guide to Muscles Function. Second Edition, Medical Science International, Tokyo, 218-225.

[31] Uchiyama, M., Lee, Y., Kazama, K., Minagawa, Y., Tsai, D.Y., Sadakata, M. and Sayama, M. (2012) Quantitative Assessment of Muscle Activity in Mammography Positioning. Proceedings of the XX IMEKO World Congress, Busan, 9-14 September 2012, TC13-P-1, 1-4.

[32] Uchiyama, M., Lee, Y., Sadakata, M., Tsai, D.Y. and Sayama, M. (2013) Effects of Mammography Positioning on the Autonomic Nervous Function. Health, 5, 1335-1341. http://dx.doi.org/10.4236/health.2013.58181

[33] Lee, Y. and Uchiyama, M. (2014) Evaluation of Physical and Psychological Burden of Subjects in Mammography. Proceedings of the IWDM World Congress, Gifu City, 29 June-2 July 2014, Vol. LNCS8539, 508-513.

[34] Uchiyama, M. (2014) The Experience of Mammography Based on the Memoirs of Examinees. Health, 6, $1310-1314$. http://dx.doi.org/10.4236/health.2014.611160 
Scientific Research Publishing (SCIRP) is one of the largest Open Access journal publishers. It is currently publishing more than 200 open access, online, peer-reviewed journals covering a wide range of academic disciplines. SCIRP serves the worldwide academic communities and contributes to the progress and application of science with its publication.

Other selected journals from SCIRP are listed as below. Submit your manuscript to us via either submit@scirp.org or Online Submission Portal.
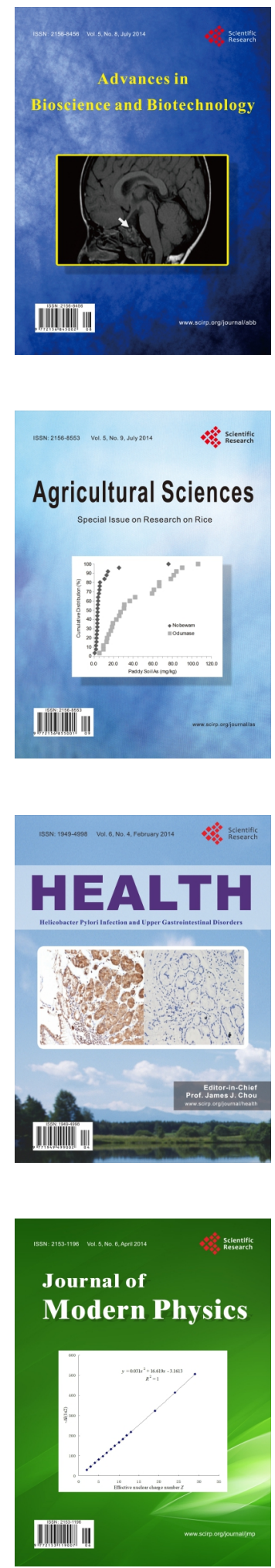
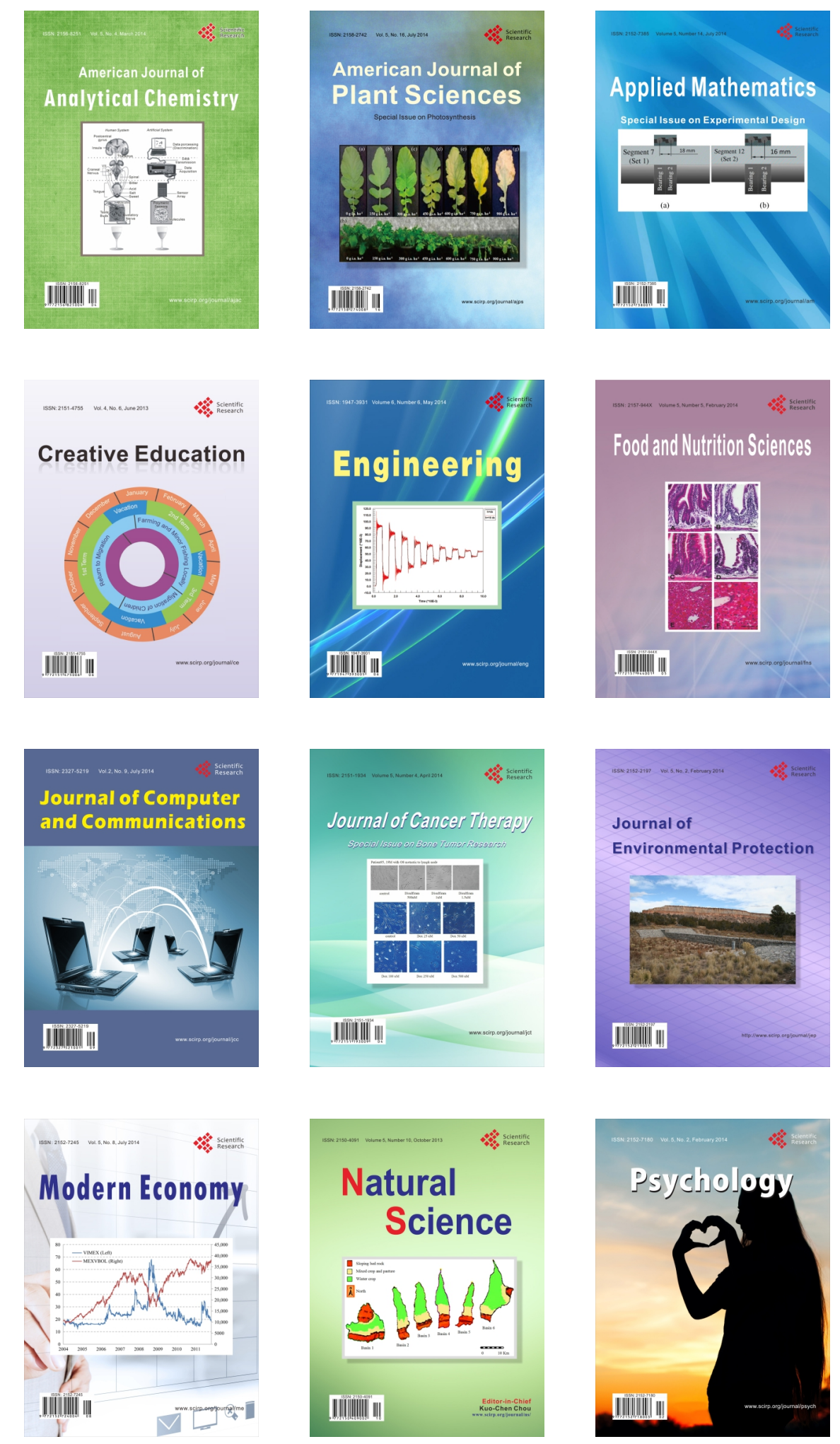